\title{
Phosphorescence of vitreous 2-bromobenzophenone
}

\author{
L.M. Buravtseva, O.S. Pyshkin, M.A. Strzhemechny, and A.A. Avdeenko \\ B. Verkin Institute for Low Temperature Physics and Engineering of the National Academy of Sciences of Ukraine \\ 47 Lenin Ave., Kharkov 61103, Ukraine \\ E-mail: buravtseva@ilt.kharkov.ua
}

Received November 13, 2007 , revised December 21, 2007

\begin{abstract}
Spectroscopic studies of vitreous 2-bromobenzophenone have been carried out over the respective domain of its stability. Glassy 2-bromobenzophenone samples were obtained by abrupt cooling of the melt by cold helium vapor. Quantum yield measurements allowed us to establish that the upper boundary of stable glass is slightly above $100 \mathrm{~K}$ while at about $220 \mathrm{~K}$ the glass melts. Phosphorescence measurements at $4.2 \mathrm{~K}$ showed that even at this low temperature the emission contains a strong excimer component. The energy position and shape (two bands) of the excimer emission are close to those observed in the crystal of 2-bromobenzophenone at higher temperatures. Contrary to findings in the crystal, the monomeric emission of the glass contains only one $\mathrm{C}=\mathrm{O}$ stretch series, every band of which is substantially broader than in the crystal. As the temperature is raised, the monomeric emission intensity goes down to completely disappear above $70 \mathrm{~K}$.
\end{abstract}

PACS: 33.50.Dq Fluorescence and phosphorescence spectra; 78.55.Qr Amorphous materials; glasses and other disordered solids.

Keywords: 2-bromobenzophenone glass, photoluminescence, quantum yield, excimer.

\section{Introduction}

Photoluminescence spectra [1] of 2-bromobenzophenone (ortho-bromobenzophenone) crystals at low temperatures in no way resemble those of any other representatives of the benzophenone family studied up to date, namely, unsubstituted benzophenone (see, for example, Melnik [2]), 44'-dichlorobenzophenone [3,4], 4-bromobenzophenone (as preliminarily quoted in our previous paper [1]). The low-temperature phosphorescence spectrum of the 2-bromobenzophenone crystal comprises very broad monomer $\mathrm{C}=\mathrm{O}$ stretch repetition bands, devoid of any traces of the rich substructure of the spectra of the other above-mentioned crystalline benzophenones, in which a coherently traveling triplet exciton emits on various traps (lattice imperfections, etc.). This gives grounds to suspect that triplet exciton transport (see the relevant review by Fayer [5]) in 2-bromobenzophenone is essentially suppressed. One way to check this inference is by measuring phosphorescence properties in the glassy state, if such exists. Luckily, in 2-bromobenzophenone it does.

Now, in order to check whether any detectable triplet exciton transport exists in 2-bromobenzophenone,we are to rely on the long-standing theory and practice [6] regarding such transport in dispersive media, especially in unsubstituted vitreous benzophenone [7-10], using time-resolved phosphorescence measurements. On the way to this ultimate goal we had to study in sufficient detail morphology relaxation as well as the optical properties of the glass state of 2-bromobenzophenone. These results constitute the subject of this paper. Detailed time-resolved phosphorescence spectra in vitreous and crystalline 2-bromobenzophenone will be published later [11].

\section{Experimental}

As previously reported [2], 2-bromobenzophenone crystallizes very reluctantly from the melt and can easily vitrify by itself with decreasing temperature. In these studies, ortho-bromobenzophenone was purified by multiple recrystallization from ethanol. Vitreous samples approximately $1.5 \mathrm{~mm}$ thick were prepared in situ as follows. A certain amount of pure crystalline matter was placed in a transparent flat quartz container with a heater wound outside at its bottom. The container was then secured in the lower chamber of a liquid helium flow cryostat. The container was heated till the crystalline sample melted, after which the container with the melt inside was cooled abruptly by helium vapor. 
Luminescence spectra were recorded using a DFS-12 automated double monochromator (linear dispersion $5.2 \AA / \mathrm{mm}$ ) and a cooled FEU-106 counter in the photon count mode. Phosphorescence spectra were corrected to account for the varying spectral sensitivity of the recording equipment. If not otherwise stated, all spectra are normalized to unity at maximum value of the respective trace. The spectral slit width did not exceed $0.5 \AA$ for all measurements. Sample excitation was performed with a LGI-21 nitrogen laser, generation wavelength $337.1 \mathrm{~nm}$, pulse duration $10 \mathrm{~ns}$, pulse sequence frequency $100 \mathrm{~Hz}$, pulse power flux $20 \mathrm{~kW} / \mathrm{cm}^{2}$. Spectra of glass samples were recorded from 4.2 to $95 \mathrm{~K}$. The temperature, maintained constant during experiments to within $\pm 1 \mathrm{~K}$ by an electronic stabilizer, was measured with a semiconductor thermometer.

Relative quantum yield measurements were performed by exciting the sample with a single pulse of the LGI-21 nitrogen laser. Registration of the total number of photons emitted by excited glassy 2-bromobenzophenone after a single excitation pulse was performed using a quantum counter based on a glycol solution of rhodamine-B.

\section{Results and discussion}

\subsection{Characterization of the vitreous state}

When working with a disordered state (like the 2-bromobenzophenone glass), which is possibly prone to spontaneous morphologic changes, it is advisable to establish the domain of its stable existence and to determine the relevant relaxation times close to and beyond that domain. To this end, several independent spectral experiments have been carried out.

First, knowing that relative quantum yield can be a sufficiently sensitive means for the above purpose we measured it as a function of temperature from 4.2 to $250 \mathrm{~K}$. The rate of temperature increase was approximately $1 \mathrm{~K} / \mathrm{min}$. The respective results are depicted in Fig. 1. In the inset we compare the non-normalized quantum yields of glassy and crystalline 2-bromobenzophenone, both curves registered under the same excitation/collection conditions. The emission of the glassy state is considerably weaker (especially, above $100 \mathrm{~K}$ ) and does not exhibit the low-temperature maximum, which is characteristic of the crystalline state. It is generally accepted $[9,13]$ that the maximum in the crystal emission can be explained as a crossover between the phonon-assisted growth of emissionless transitions at higher temperatures and a similar quenching owing to traps at lower temperatures when the role of triplet exciton transport increases. A blowup of the normalized lower curve for the glassy state in the inset is shown in the main plot of Fig. 1. It is clearly seen that there are two «critical» points where the sample morphology suffers changes. One point labeled 1

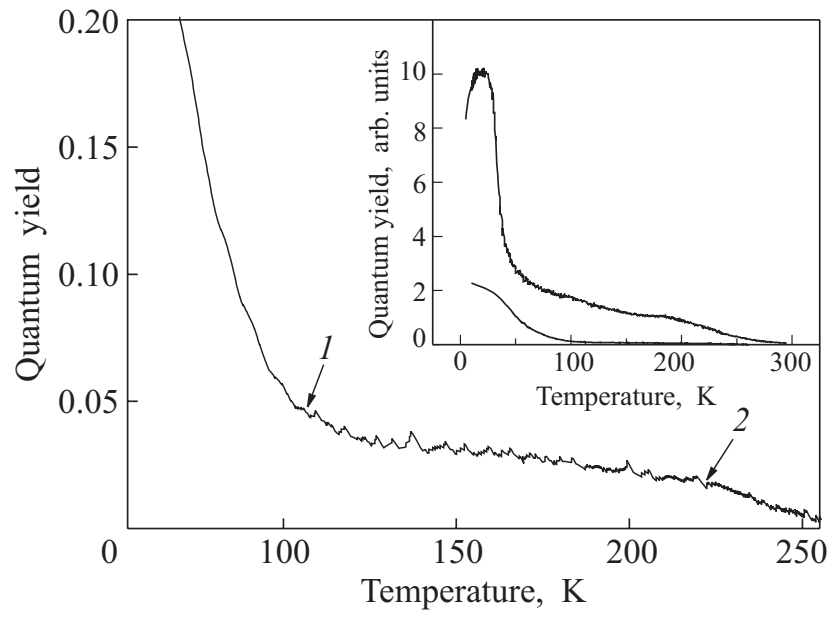

Fig. 1. Temperature dependence of the relative quantum yield of glassy 2-bromobenzophenone. The inset: comparison of the quantum yields of the glassy (lower trace) and crystalline (upper trace) samples.

(at about $100 \mathrm{~K}$ ) can be treated as the temperature where the glass looses its stability, undergoing some morphologic changes. This process stops at point 2 (about $220 \mathrm{~K}$ ), above which the appearing state is the liquid state, which could be observed with the naked eye. This critical point is the vitrification/liquification temperature. As the temperature grew further to $300 \mathrm{~K}$, the sample did not crystallize. Even at room temperature it remained liquid for quite a long time (see next paragraph). Such behavior is indirect evidence that our 2-bromobenzophenone sample was highly pure. A similar temperature history was observed by Babkov et al. [14], except for the difference that their sample crystallized uneventfully around $300 \mathrm{~K}$.

Second, the vicinity around the critical point 1 in Fig. 1 was checked for long-time stability. In Fig. 2 we show how the phosphorescence spectrum of the initially glassy state changed during about $20 \mathrm{~h}$ at a fixed temperature of $95 \mathrm{~K}$. The spectrum of a fresh vitreous sample (trace 1 ) is a very broad structureless band. Compared to a similar spectrum from a neat 2-bromobenzophenone crystal (curve 4), this band is positioned approximately within the same range. Knowing that the crystal spectrum at this temperature [1] is a superposition of the monomer and excimer emissions, we may conclude that the glass spectrum either does not contain appreciable monomeric components or they are inhomogeneously smeared so that the monomer $\mathrm{C}=\mathrm{O}$ stretch series cannot be resolved. After $20 \mathrm{~h}$ the spectrum (curve 2) retained its primary bell-like shape without distinct features, its center having appreciably shifted to blue during the waiting time. It is noteworthy that this shift is in the opposite direction relative to the phosphorescence spectrum (curve 4) of the crystal 


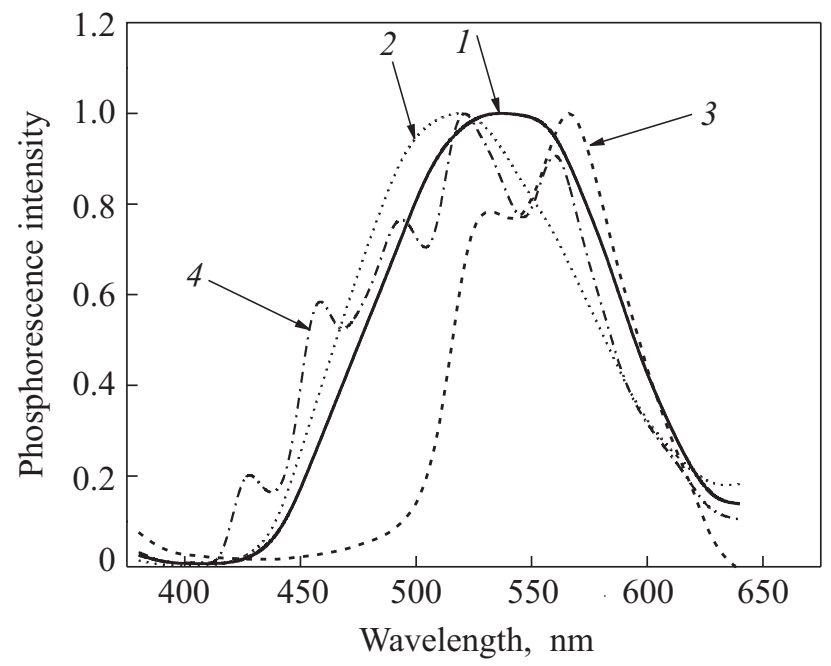

Fig. 2. Evolution of the phosphorescence spectrum of 2-bromobenzophenone in time at a fixed temperature. The solid trace 1 is for a freshly prepared glass sample warmed up to $95 \mathrm{~K}$; the dotted trace 2 is for the same sample kept for more than $20 \mathrm{~h}$ at $T=95 \mathrm{~K}$; the dashed trace 3 is for the same sample after it was kept at room temperature for about $168 \mathrm{~h}$. The spectrum protect [1] from a neat crystal of 2-bromobenzophenone at $95 \mathrm{~K}$ is shown for comparison as the dash-curve dot 4 .

at the same temperature [1]. This shift suggests certain changes in morphology but, possibly, not closer to the crystalline order. Warmed up to room temperature and kept so sufficiently long (about $168 \mathrm{~h}$ ), the sample exhibited a nice double-hump spectrum, characteristic of excimer emission and virtually coincident with the one obtained from the crystal.

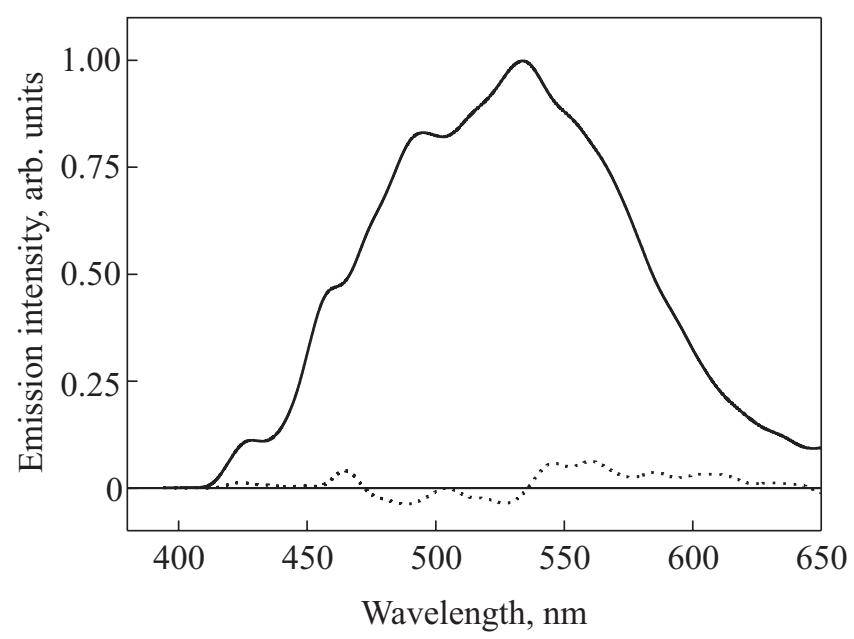

Fig. 3. The phosphorescence spectrum from a freshly prepared vitreous 2-bromobenzophenone at $75 \mathrm{~K}$ (upper solid curve) and the difference (lower dotted curve) between this spectrum and the one obtained after the sample was cooled down to $4.2 \mathrm{~K}$, kept thus for three hours, and re-warmed back to $75 \mathrm{~K}$.
Third, in order to check whether the glass state within its domain of stability undergoes any essential relaxation-related changes with time, we measured phosphorescence spectra (see the bell-shaped curve in Fig. 3) of vitreous 2-bromobenzophenone at $75 \mathrm{~K}$, i.e., well inside the domain of existence of the glass. Then the sample was cooled down to liquid helium temperature and after a three hour waiting was warmed up back to $75 \mathrm{~K}$. After that we again measured the spectrum. The difference between this spectrum and the initial one (solid curve) is shown as the dotted curve in Fig. 3. Thus, even at a quite high temperature the spectrum is well reproduced after certain manipulations.

\subsection{Temperature dependence of phosphorescence spectra}

Let us first discuss the spectrum of vitreous 2-bromobenzophenone at low temperatures where effects of phonon-assisted spectral kinetics are minimal. In order to demonstrate the peculiarities of low-temperature glass spectra, in Fig. 4 we compare them with similar spectra of the crystal [1]. The glass spectrum has much in common with the respective spectrum of the crystal but with considerable or even cardinal differences. The low-temperature glass spectrum in Fig. 4 consists of several broad bands separated in energy by the stretch mode frequency of the $\mathrm{C}=\mathrm{O}$ bond (about $1700 \mathrm{~cm}^{-1}$ ). All monomer bands of the glass at low temperatures are broader even compared with the unusually broad bands in the crystal phosphorescence, which can be naturally attributed to a larger inhomogeneous broadening in the glass. Being clearly

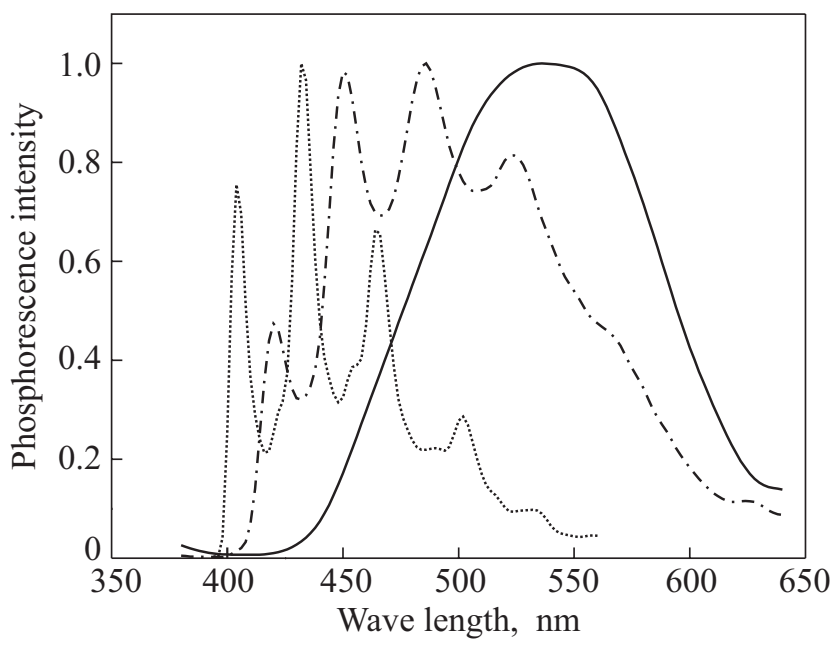

Fig. 4. Phosphorescence spectra from a freshly prepared glass-like 2-bromobenzophenone at $4.2 \mathrm{~K}$ (dot-dash line) and $111 \mathrm{~K}$ (solid line). The low-temperature spectrum $(1.6 \mathrm{~K})$ of 2-bromobenzophenone crystal protect[1] is shown as a dotted line for comparison. 
monomeric, this series is nevertheless superimposed on a broad feature at the red side, which can be attributed as belonging to excimer emission [15]. This first important difference can be tentatively explained by arguing that in a glass, unlike in the regular crystal, there are mutual positions of molecules with minimal or no energy barriers that could preclude excimer formation. Another essential difference between the spectra of vitreous and crystalline 2-bromobenzophenone is that only one set of equidistant $\mathrm{C}=\mathrm{O}$ stretch bands is present in the glass spectrum, rather than two, as was reported previously for crystals [1]. We remind here that the two equidistant sets in the crystal were ascribed to the emission from two excited states (stable and metastable ones), separated by approximately $1100 \mathrm{~cm}^{-1}$ with an energy barrier of approximately $100 \mathrm{~cm}^{-1}$ between them, which can be overcome via thermal activation approximately at and above the liquid nitrogen temperature. Keeping to that interpretation, the emission in the glass state of 2-bromobenzophenone occurs from the global minimum of the triplet excited molecular state. This implies that the excited molecule in the glass environment can easily find the pathway from the excited (primarily metastable) state to the global minimum, where it emits to $\mathrm{C}=\mathrm{O}$ vibrational sublevels of the ground state.

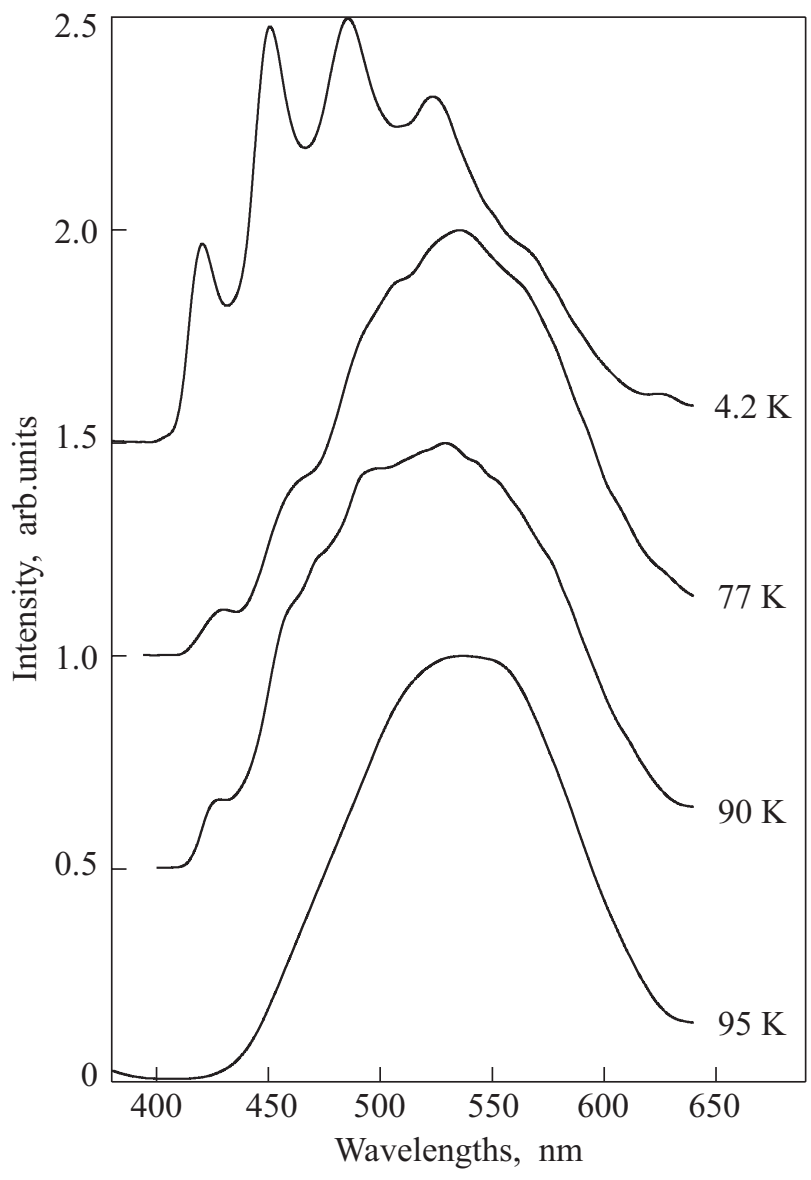

Fig. 5. Phosphorescence spectra from a freshly prepared glass-like 2-bromobenzophenone at four temperatures.

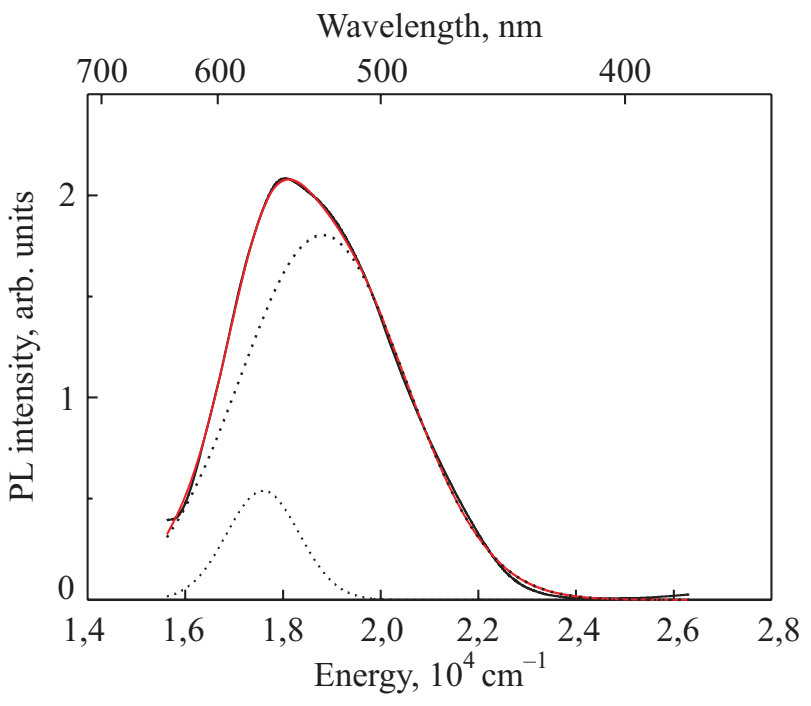

Fig. 6. The phosphorescence spectrum of vitreous 2-bromobenzophenone at $95 \mathrm{~K}$ resolves nicely into two Gaussian-shaped subbands. The total virtually coincides with the experimental trace.

Variations of phosphorescence spectra of 2-bromobenzophenone glass with temperature are shown in Fig. 5. At the lowest temperature of $4.2 \mathrm{~K}$, the spectrum is to a large extent monomeric. However, the spectrum clearly contains a structureless very broad background band. As the temperature was raised, we observed continuous changes of the phosphorescence spectrum. Compared to the low-temperature spectrum, the spectra at intermediate temperatures within the domain of stability shift with temperature progressively to red. The predominant feature is the very broad structureless maximum, with only a host of the monomer emission series remaining. This predominant feature was shown to be due to a triplet excimer [15]. Indeed, this very broad band appears in the glass within the same wavelength range as in the crystal [16] with the difference that in the glass it does not clearly resolve into two components. By analogy, we conclude that to a high degree of reliability this broad band in the phosphorescence spectrum of the glass is also of excimer origin. The sole broad band in the phosphorescence of vitreous 2-bromobenzophenone at higher temperatures (close to $100 \mathrm{~K}$ ) is clearly asymmetric. It can be resolved into two Gaussian-shaped components as shown in Fig. 6. As regards the position and the relative intensities of these two components they are very much close to those observed in the crystalline 2-bromobenzophenone.

\section{Conclusions}

The glass state of 2-bromobenzophenone was characterized, using its relative phosphorescence quantum yield, in order to establish its domain of stable existence as well as the typical rates of morphologic changes. The 
critical points at which the morphology suffered basic transformations are at 100 and $220 \mathrm{~K}$.

Phosphorescence spectra in vitreous 2-bromobenzophenone have been measured as a function of temperature over the entire domain of existence of the glassy state. These spectra are compared with similar spectra of crystalline 2-bromobenzophenone. Unlike in the crystal, triplet excimers are formed at low temperatures. The excimer quantum yield even at the lowest temperature exceeds that of the monomer emission. This finding is tentatively explained as being due to lesser steric restrictions for an excited molecule to accommodate itself in its surrounding, as compared to the crystal, in order to form a bimolecular excimer.

The monomeric emission comprises only one $\mathrm{C}=\mathrm{O}$ stretch series, which corresponds to the transitions from the presumably global energy minimum [1] of an ultimately relaxed excited molecule. This observation can also find its explanation in the higher degree of conformational freedom of the molecule in the glass.

Though phosphorescence spectra of glassy 2-bromobenzophenone helps understand the nature of phosphorescence emission, time-resolved phosphorescence experiments are needed to get a better insight into the main mechanisms that control the spectroscopic effects observed.

\section{Acknowledgments}

The authors sincerely appreciate Yu.M. Strzhemechny's informational support. The authors also thank N.A. Davydova for a preprint of her paper [14] prior to publication as well as P.V. Zinoviev for his critical reading of the manuscript.
1. A.A. Avdeenko, O.S. Pyshkin, V.V. Eremenko, M.A. Strzhemechny, L.M. Buravtseva, and R.V. Romashkin, Fiz. Nizk. Temp. 32, 1355 (2006) [Low Temp. Phys. 32, 1028 (2006)].

2. V.I. Melnik, Spectral Luminescence Studies of Triplet States in Molecular Systems, Dr. Sci. Thesis, Institute of Physics, Kiev (2006).

3. A.A. Avdeenko, T.L. Dobrovolskaya, V.A. Kulchitskii, and Yu.V. Naboikin, Phys. Status Solidi B90, 405 (1980).

4. A.A. Avdeenko, L.M. Buravtseva, V.S. Gorobchenko, V.V. Eremenko, S.V. Izvekov, A.E. Kravchenko, O.S. Pyshkin, and V.I. Sugakov, Fiz. Nizk. Temp. 23, 334 (1997) [Low Temp. Phys. 23, 247 (1997)].

5. M.D. Fayer, Exciton Coherence, in: Spectroscopy and Excitation Dynamics of Condensed Molecular Systems, V.M. Agranovich and R.M. Hochstrasser (eds.), North-Holland, Amsterdam (1983), p.185.

6. H. Bässler, in: Disorder Effects in Relaxational Processes R. Richert and A. Blumen (eds.), Springer-Verlag (1994), p. 485 .

7. R. Richert and H. B »assler, Chem. Phys. Lett. 118, 235 (1985).

8. R. Richert and H. Bässler, J. Chem. Phys. 84, 3567 (1985).

9. V.I. Melnik, Fiz. Tverd. Tela 40, 1052 (1998).

10. N.A. Davydova, V.I. Mel'nik, K.I. Nelipovitch, and M. Drozd, J. Mol. Struct. 555, 187 (2000).

11. O.S. Pyshkin, L.M. Buravtseva, and M.A. Strzhemechny (in prepartion).

12. V.N. Baumer, R.V. Romashkin, M.A. Strzhemechny, A.A. Avdeenko, O.S. Pyshkin, R.I. Zubatyuk, and L.M. Buravtseva, Acta Crystallogr. E61, o1170 (2005).

13. S.W. Mao and N. Hirota, Molec. Phys. 27, 327 (1974).

14. L.M. Babkov, J. Baran, N.A. Davydova, D. Drozd, O.S. Pyshkin, and K.E. Uspenskiy (submitted to J. Mol. Struct.).

15. O.S. Pyshkin, Fiz. Nizk. Temp. 33, 1411 (2007) [Low Temp. Phys. 33, 1077 (2007)].

16. M.A. Strzhemechny, A.A. Avdeenko, V.V. Eremenko, O.S. Pyshkin, and L.M. Buravtseva, Chem. Phys. Lett. 431, 300 (2006). 